\title{
Risk Factors and Clinical Outcomes of Cognitive Impairment in Diabetic Patients Undergoing Peritoneal Dialysis
}

\author{
Xuan Huang ${ }^{a, b}$ Chunyan $Y^{a, b}$ Meiju Wu ${ }^{a, b}$ Yagui Qiua,b Haishan $W^{a, b}$ \\ Hongjian $Y^{a, b}$ Yuan Peng ${ }^{a, b} \quad X_{i} X_{i a o^{a, b}}$ Jianxiong Lin ${ }^{a, b}$ Xuqing $Y^{a, b}$ \\ Xiao Yang ${ }^{a, b}$

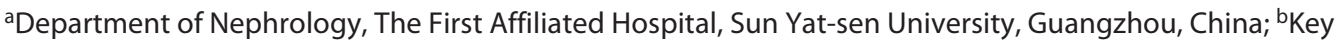 \\ Laboratory of Nephrology, National Health Commission and Guangdong Province, Guangzhou, China
}

\section{Keywords}

Cognitive dysfunction · Diabetes mellitus · Peritoneal dialysis

\begin{abstract}
Introduction: Cognitive impairment $(\mathrm{Cl})$ is common in patients with CKD or diabetes mellitus (DM). However, the relevance between $\mathrm{DM}$ and $\mathrm{Cl}$ in diabetic patients undergoing peritoneal dialysis (PD) has not been clearly established. This study aimed to explore the role of $\mathrm{DM}$ in $\mathrm{Cl}$, the association of glycemic control with $\mathrm{Cl}$, and clinical outcomes of $\mathrm{Cl}$ in diabetic PD patients. Methods: Continuous ambulatory PD (CAPD) patients followed up in our PD center between 2014 and 2016 were enrolled. The participants were followed until an endpoint was reached or December 2017. Demographic data and clinical characteristics were collected, and laboratory parameters were measured. The Montreal Cognitive Assessment (MoCA) was used to evaluate global cognitive function, and a score of $<26$ was considered to indicate $\mathrm{Cl}$. A propensity score matching according to age, gender, and mean arterial pressure was conducted between the DM and
\end{abstract}

non-DM groups. Results: A total of 913 CAPD patients were enrolled, of whom 186 (20.4\%) had diabetes. After appropriate matching, 175 patients in the DM group and 270 patients in the non-DM group were included. Patients with diabetes had a higher prevalence of $\mathrm{Cl}$ and lower scores for visuospatial/executive function, naming, language, delayed recall, and orientation. Higher $\mathrm{HbA} 1 \mathrm{c}$ (odds ratio [OR], 1.547; 95\% confidence interval $[95 \% \mathrm{Cl}], 1.013-2.362)$ and cardiovascular disease (CVD; OR, 2.926; 95\% Cl, 1.139-7.516) significantly correlated with a risk of $\mathrm{Cl}$ in diabetic patients. During a median of 26.0 (interquartile range 13.5-35.6) months of follow-up, diabetic patients with $\mathrm{Cl}$ demonstrated a significantly lower survival rate than those without $\mathrm{Cl}$, and $\mathrm{Cl}$ was an independent risk factor for mortality after adjustment (hazard ratio, $7.224 ; 95 \% \mathrm{Cl}, 1.694-30.806$ ). However, they did not show worse technique survival or higher peritonitis rate than patients without $\mathrm{Cl}$. Conclusions: $\mathrm{HbA} 1 \mathrm{c}$ and CVD are independent risk factors for $\mathrm{Cl}$ in diabetic patients undergoing CAPD, and $\mathrm{Cl}$ is independently associated with a higher risk of mortality.

(c) 2021 The Author(s) Published by S. Karger AG, Basel
(C) 2021 The Author(s)

Published by S. Karger AG, Basel

This article is licensed under the Creative Commons AttributionNonCommercial-NoDerivatives 4.0 International License (CC BYNC-ND) (http://www.karger.com/Services/OpenAccessLicense) Usage and distribution for commercial purposes as well as any distribution of modified material requires written permission.
Correspondence to:

Xiao Yang, yangxsysu@ 126.com 


\section{Introduction}

Cognitive impairment (CI) is a common finding in patients with CKD and has been shown to correlate with the severity of renal insufficiency [1]. It has been reported that $17-62 \%$ of CKD patients exhibit impairments in cognitive performance [1-3], and this number is even higher among patients with ESRD [4-6]. Cognitive performance includes multiple domains, including memory, attention, information processing, executive functioning, visuospatial skills, and language abilities. Due to the fact that peritoneal dialysis (PD) involves complex medical regimens, impairment in cognitive function may interfere with patients' abilities of decision-making and self-care, implementation of fluid and dietary restriction, and adherence to medication instructions. In addition, $\mathrm{CI}$ is associated with dialysis withdrawal [7], a higher hospitalization rate, higher mortality, and a poorer quality of life $[8,9]$. Moreover, CI may considerably increase the overall disease burden and healthcare expenditure.

As the leading cause of ESRD in the USA, diabetes mellitus (DM) accounts for 38\% of patients with ESRD according to the US Renal Data System (USRDS) [10], and in our PD center, the prevalence of diabetes is $\sim 20-$ $25 \%[11,12]$. Substantial epidemiologic evidence has established an association between DM and cognitive dysfunction [13, 14], which is particularly strong in older populations. Furthermore, the prognosis of $\mathrm{CI}$ is worse in patients with diabetes than in those without $[15,16]$. However, the relevance between DM and CI in patients undergoing PD has not been clearly established. Therefore, in the present study, we aimed to explore whether the impact of DM on CI changes in patients undergoing $\mathrm{PD}$, the association of glycemic control with CI, and clinical outcomes of $\mathrm{CI}$ in diabetic PD patients.

\section{Materials and Methods}

\section{Study Population and Data Collection}

The continuous ambulatory PD (CAPD) patients from the First Affiliated Hospital of Sun Yat-sen University were consecutively recruited between January 1, 2014, and December 31, 2016. The eligible patients were $\geq 18$ years old and had been undergoing CAPD treatment for $\geq 30$ days. Patients who were unable to complete cognitive testing due to pre-existing dementia, mental disorder, severe eyesight loss, illiteracy, or a formal education of $<6$ years were excluded from the study. The participants were followed until death, transfer to hemodialysis (HD), kidney transplantation, transfer to another center, or December 31, 2017. The study was conducted in compliance with the ethical principles of the Helsinki Declaration and approved by the Human Ethics Com- mittee of Sun Yat-sen University. Written informed consent was obtained from all the participants.

Demographic and clinical data were collected that included age, gender, level of education, BMI, the histories of smoking, diabetes, hypertension, cardiovascular disease (CVD), the duration of dialysis, and the use of erythropoietin. Hypertension was defined when a systolic blood pressure (SBP) $\geq 140 \mathrm{~mm} \mathrm{Hg}$ and/or a diastolic blood pressure $\geq 90 \mathrm{~mm} \mathrm{Hg}$. CVD was defined as a history of coronary heart disease, heart failure, cerebrovascular disease, peripheral artery disease, or other types of vascular diseases [11]. Cerebrovascular disease was recorded separately. Laboratory data including $\mathrm{Kt} / \mathrm{V}$, measured glomerular filtration rate (mGFR), normalized protein catabolic rate, SBP, diastolic blood pressure, mean arterial pressure (MAP), hemoglobin concentration, hematocrit, high-sensitivity C-reactive protein (hsCRP), serum albumin, serum calcium, serum phosphorus, intact parathyroid hormone, serum sodium, serum potassium, BUN, serum Cr, serum uric acid, serum lipid, serum glucose, and $\mathrm{HbAlc}$ were also measured. mGFR was measured as the mean of urea and $\mathrm{Cr}$ clearance calculated from 24-h urine collections and adjusted for the body surface area. The normalized protein catabolic rate is a nutritional indicator used to estimate protein intake. It was calculated from 24-h urine and dialysate effluent collections and normalized to ideal body weight. The weekly total Kt/V (urea clearance index) was calculated from 24-h urine and dialysate effluent collections. The volume of distribution of urea was estimated from the Watson formula [17].

\section{Measurement of Cognitive Function}

Participants were assessed using the Chinese mandarin version of Montreal Cognitive Assessment (MoCA) for global cognition. Cognitive dysfunction refers to a deficit of key brain functions in one or more cognitive domains. It ranges from mild cognitive impairment to severe dementia [18]. The MoCA is a rapid screening instrument for mild cognitive dysfunction, which is considered as a prodromal stage of dementia. It can assess different domains of cognitive function, including attention and concentration, executive functions, memory, language, visuoconstructional skills, conceptual thinking, calculations, and orientation. Compared to other cognitive screening tools, such as the Mini-Mental State Examination, the clock-drawing test, Memory Impairment Screen, Mental Status Questionnaire, Mini-Cog verbal fluency, 8-Item Informant Interview, Functional Activities Questionnaire, 7-Minute Screen, and Abbreviated Mental Test, the MoCA is easy to administer and has been validated to be more or equally sensitive [19-21]. The highest possible score is 30 points, and a score below 26 is considered cognitively impaired. Those who received $\leq 12$ years of formal education are given 1 additional point (www.mocatest.org). It took approximately $10 \mathrm{~min}$ to administer the MoCA. The assessment was performed by $\mathrm{PD}$ nurses receiving standardized training during the patients' regular monthly visits, since it would be more convenient for the PD nurses to perform assessment if including MoCA in the routine screening. But a full diagnosis of CI still needs further confirmation by a specialist after screening.

\section{Statistical Analysis}

Categorical variables are expressed as frequencies and percentages, and continuous variables are expressed as means \pm standard deviations (SDs) for normally distributed data and medians (interquartile ranges [IQRs]) for nonnormally distributed data. For 
Fig. 1. Flowchart of patient selection. PD, peritoneal dialysis.

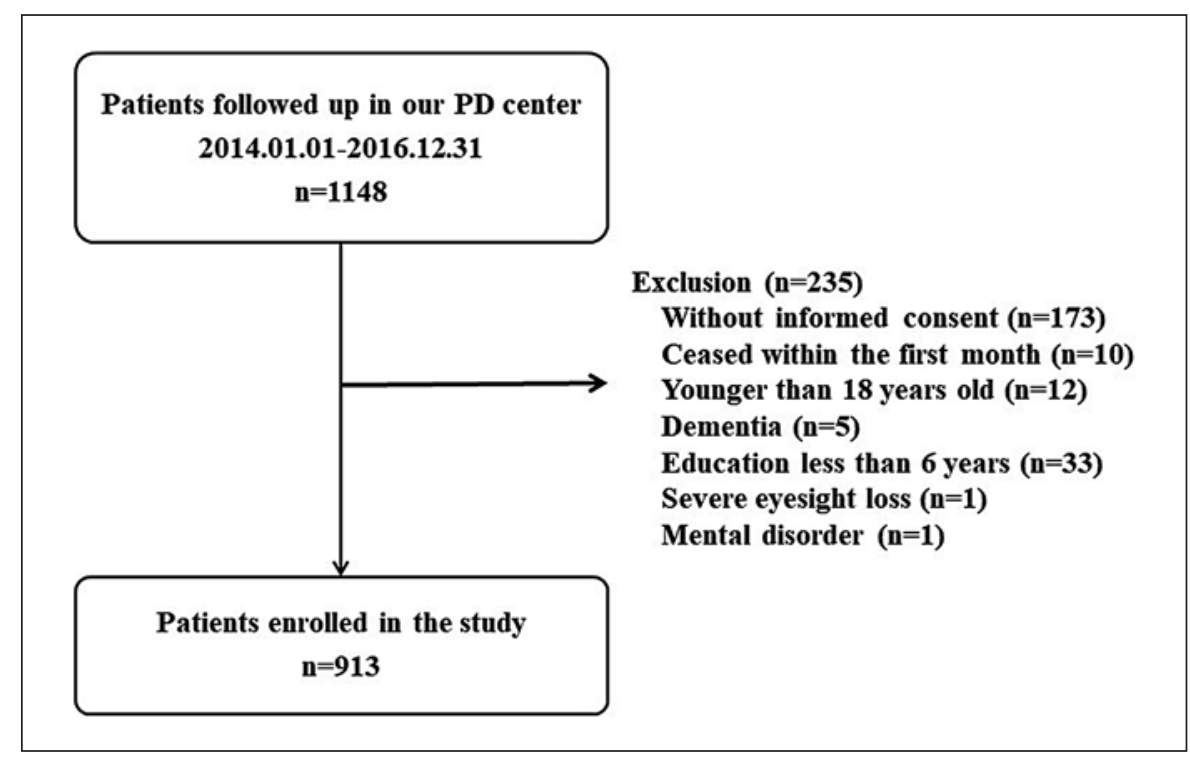

comparisons between groups, independent sample $t$-tests were performed for normally distributed variables, Mann-Whitney $U$ tests were used to compare nonnormally distributed variables, and the $\chi^{2}$-tests were applied for categorical variables. To compensate for the differences between the DM and non-DM groups, a propensity score was calculated using a logistic regression with age, gender, and MAP. Patients in the DM group were matched at a ratio of 1:2 with those in the non-DM group by individual propensity scores. Patients who were successfully matched were selected for the following analyses. The associations between DM and CI were examined by multivariable logistic regression models. Variables with $p<0.1$ in the univariate analysis were selected for the multivariate adjusted model. The final full model included age, gender, level of education, history of diabetes, history of CVD, dialysis vintage, SBP, serum $\mathrm{Cr}$, and high-density lipoprotein cholesterol (HDL-C). To identify the risk factors related to CI in diabetic patients, a multivariable logistic regression model including age, gender, education, BMI, history of smoking, CVD, MAP, total cholesterol, serum $\mathrm{Cr}$, glucose, or $\mathrm{HbA1c}$ was used. The results are presented as odds ratios (ORs) and 95\% confidence intervals (95\% CIs). The relationships of CI with patient survival and technique survival were determined using Kaplan-Meier estimates, and the relationship between CI and the incidence of peritonitis was assessed using a Poisson regression analysis. After univariate analyses, multivariate Cox proportional hazards regression was calculated to determine the risk of mortality. The results are presented as hazard ratios (HRs) and 95\% CIs. A p-value of less than 0.05 was considered statistically significant. All statistical analyses were carried out using SPSS version 24.0 (SPSS Inc., Armonk, NY, USA).

\section{Results}

\section{Patient Characteristics}

Of the 1,148 CAPD patients who attended the unit during the study period, 173 did not give informed con- sent, 10 were on CAPD for $<1$ month, 12 were $<18$ years old, and 40 were unable to complete cognitive testing due to dementia ( 5 patients), a formal education of $<6$ years (33 patients), severe eyesight loss (1 patient), or mental disorder (1 patient). Finally, the remaining 913 patients were enrolled in this study (Fig. 1). Their mean $( \pm$ SD) age was $48.7 \pm 14.6$ years; $60.4 \%(n=551)$ were male, and $20.4 \%(n=186)$ had diabetes. The median duration of PD was 25.5 (IQR 6.0-52.6) months, and the mean BMI was 21.9 (IQR $19.8-24.2) \mathrm{kg} / \mathrm{m}^{2}$. Of these patients, $92.8 \% \mathrm{had}$ a history of hypertension and $22.3 \%$ had a history of CVD. The 186 diabetic patients had a mean $( \pm S D)$ age of $60.8 \pm 11.0$ years, and $68.8 \%(n=128)$ were male. Overall, $97.8 \%$ of them had a history of hypertension and $47.3 \%$ had a history of CVD. Compared with the nondiabetic patients, diabetic patients in this study were older and more often men. They also had higher BMI, a higher prevalence of hypertension and CVD, a higher proportion of assisted PD, a higher level of hsCRP, triglyceride, serum potassium, blood glucose, and $\mathrm{HbA1c}$ but shorter dialysis vintage, a lower level of MAP, serum albumin, calcium, phosphate, Cr, low-density lipoprotein cholesterol, and HDL-C. There were no significant differences between groups in mGFR, Kt/V, hemoglobin, serum sodium, BUN, uric acid, and total cholesterol (Table 1 and see online suppl. Fig. 1; for all online suppl. material, see www.karger.com/doi/10.1159/000514172).

After propensity score matching according to age, gender, and MAP, 175 of the 186 patients in the DM group and 270 of the 727 patients in the non-DM group were matched. No significant differences were observed 


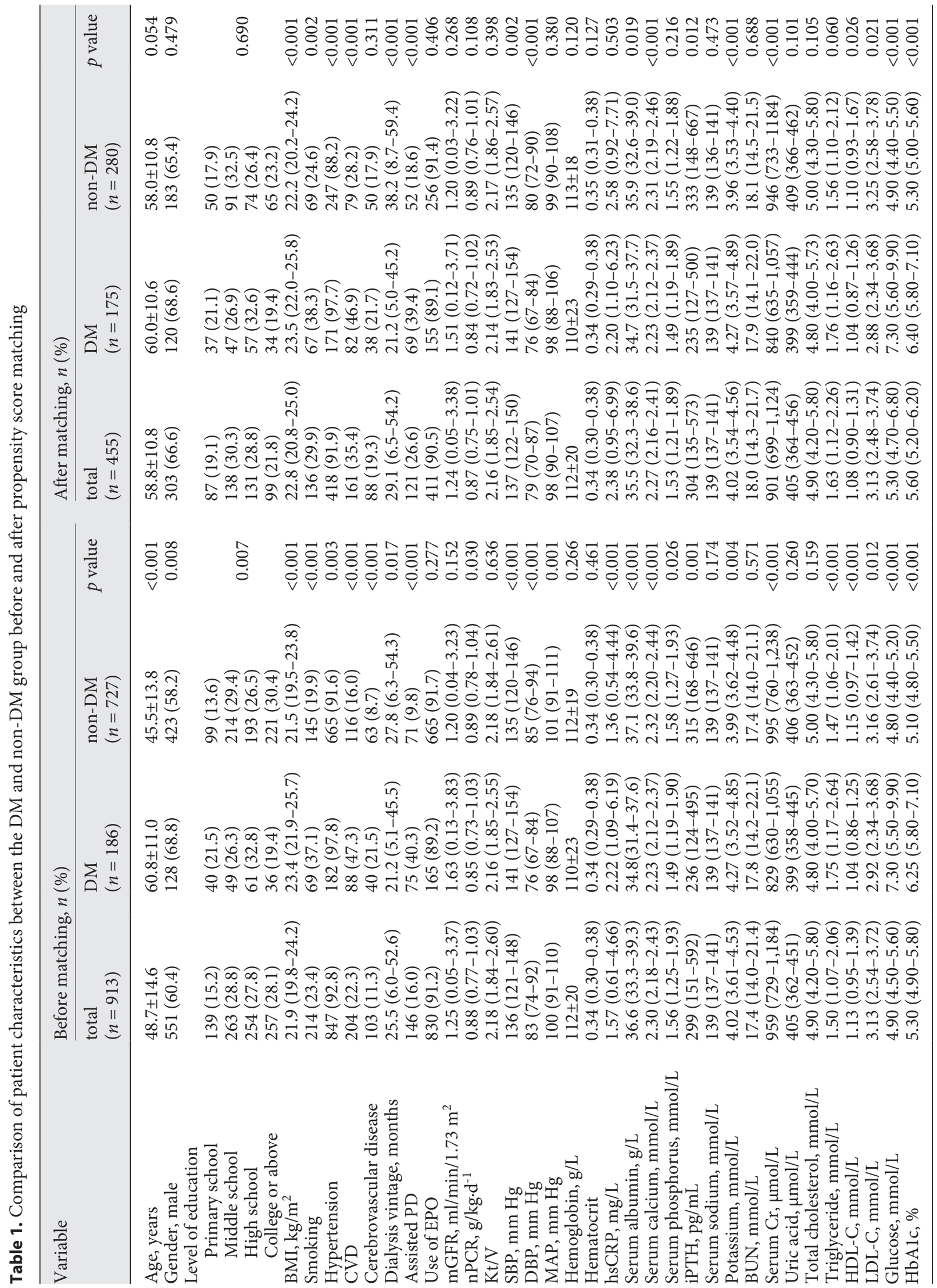

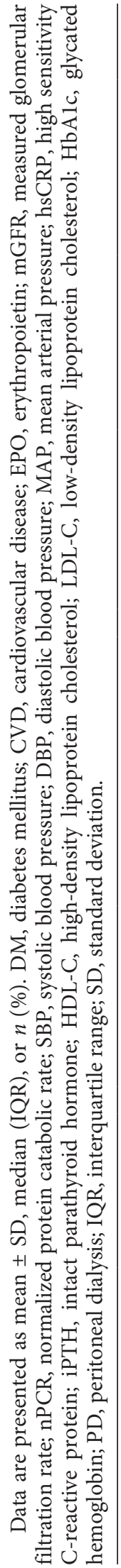


Table 2. Prevalence of cognitive impairment and MoCA score between patients with and without DM

\begin{tabular}{llllrr}
\hline & Score & Total & DM & Non-DM & $p$ value \\
\hline Cognitive impairment, $\%$ & - & $331(72.7)$ & $141(80.6)$ & $190(67.9)$ & 0.003 \\
Total score & 30 & $22.0 \pm 4.7$ & $20.7 \pm 4.9$ & $22.9 \pm 4.4$ & $<0.001$ \\
Visuospatial/executive & 5 & $3.47 \pm 1.32$ & $3.10 \pm 1.34$ & $3.71 \pm 1.25$ & $<0.001$ \\
Naming & 3 & $2.39 \pm 0.86$ & $2.24 \pm 0.95$ & $2.48 \pm 0.78$ & 0.003 \\
Attention & 6 & $5.17 \pm 1.03$ & $5.05 \pm 1.10$ & $5.24 \pm 0.99$ & 0.055 \\
Language & 3 & $1.90 \pm 0.84$ & $1.77 \pm 0.86$ & $1.99 \pm 0.81$ & $<0.006$ \\
Abstraction & 2 & $0.00(0.00-1.00)$ & $0.00(0.00-1.00)$ & $0.00(0.00-1.00)$ & 0.130 \\
Delayed recall & 5 & $2.37 \pm 1.75$ & $2.03 \pm 1.74$ & $2.59 \pm 1.73$ & 0.001 \\
Orientation & 6 & $5.73 \pm 0.70$ & $5.57 \pm 0.89$ & $5.83 \pm 0.52$ & $<0.001$ \\
\hline
\end{tabular}

Data are presented as mean $\pm \mathrm{SD}$, median (IQR), or $n(\%)$. DM, diabetes mellitus; IQR, interquartile range; $\mathrm{SD}$, standard deviation.

Table 3. Associated factors for cognitive impairment in diabetic patients

\begin{tabular}{|c|c|c|c|c|c|c|}
\hline \multirow[t]{2}{*}{ Variable } & \multicolumn{2}{|l|}{ Unadjusted } & \multicolumn{2}{|l|}{ Model 1} & \multicolumn{2}{|l|}{ Model 2} \\
\hline & OR (95\% CI) & $p$ value & OR (95\% CI) & $p$ value & OR (95\% CI) & $p$ value \\
\hline Gender, male & $0.502(0.204-1.237)$ & 0.134 & $0.568(0.189-1.708)$ & 0.314 & $0.478(0.157-1.457)$ & 0.194 \\
\hline 12 -year education & $0.610(0.284-1.313)$ & 0.207 & $0.715(0.313-1.630)$ & 0.425 & $0.671(0.293-1.540)$ & 0.347 \\
\hline BMI, $\mathrm{kg} / \mathrm{m}^{2}$ & $0.934(0.825-1.058)$ & 0.282 & $0.899(0.767-1.053)$ & 0.187 & $0.874(0.743-1.028)$ & 0.105 \\
\hline MAP, mm Hg & $1.007(0.980-1.035)$ & 0.594 & $1.021(0.990-1.053)$ & 0.194 & $1.025(0.993-1.059)$ & 0.128 \\
\hline Serum Cr, $\mu \mathrm{mol} / \mathrm{L}$ & $0.999(0.998-1.000)$ & 0.182 & $1.000(0.999-1.002)$ & 0.800 & $1.000(0.999-1.002)$ & 0.787 \\
\hline Total cholesterol, mmol/L & $1.314(0.951-1.816)$ & 0.098 & $1.274(0.890-1.823)$ & 0.185 & $1.354(0.945-1.939)$ & 0.098 \\
\hline Glucose, $\mathrm{mmol} / \mathrm{L}$ & $1.144(1.006-1.301)$ & 0.040 & $1.122(0.974-1.293)$ & 0.111 & - & - \\
\hline HbAlc, $\%$ & $1.397(0.981-1.991)$ & 0.064 & - & - & $1.547(1.013-2.362)$ & 0.043 \\
\hline
\end{tabular}

OR, odds ratio; 95\% CI, 95\% confidence interval; CVD, cardiovascular disease; MAP, mean arterial pressure; HbA1c, glycated hemoglobin.

between the 2 groups in age, gender, and MAP. They had comparable education level, mGFR, hsCRP, Kt/V, hemoglobin, serum phosphate, serum sodium, BUN, uric acid, total cholesterol, and triglyceride levels. 455 patients after matching had a mean $( \pm S D)$ age of $58.8 \pm 10.8$ years and $66.6 \%(n=303)$ were male. The median duration of PD was 29.1 (IQR 6.5-54.2) months. Among them, 91.9\% had a history of hypertension and $35.4 \%$ had a history of $\mathrm{CVD}$. The mean $( \pm \mathrm{SD})$ age of the diabetic patients was $60.0 \pm 10.6$ years and $68.6 \%(n=120)$ were male (Table 1$)$.

\section{DM and Cognitive Impairment}

The prevalence of CI was $72.7 \%(n=331)$ in the matched population, and the mean $( \pm \mathrm{SD})$ MoCA score was $22.0 \pm 4.7$ points. The patients with DM had a sig- nificantly higher prevalence of CI $(80.6 \%)$ than those without (67.9\%). Furthermore, they had significantly lower scores for visuospatial/executive function (3.10 \pm 1.34 vs. $3.71 \pm 1.25$ points), naming ( $2.24 \pm 0.95$ vs. 2.48 \pm 0.78 points $)$, language $(1.77 \pm 0.86$ vs. $1.99 \pm 0.81$ points), delayed recall ( $2.03 \pm 1.74$ vs. $2.59 \pm 1.73$ points $)$, and orientation ( $5.57 \pm 0.89$ vs. $5.83 \pm 0.52$ points) (Table 2). The association between DM and CI was significant by univariate logistic analysis (OR, 1.964; 95\% CI, 1.252-3.083). After adjusting for confounders, DM was associated with a 1.677-fold higher risk of CI (online suppl. Table 1). No clear correlation was found between the hemoglobin level and the MoCA score (online suppl. Fig. 2). 
A

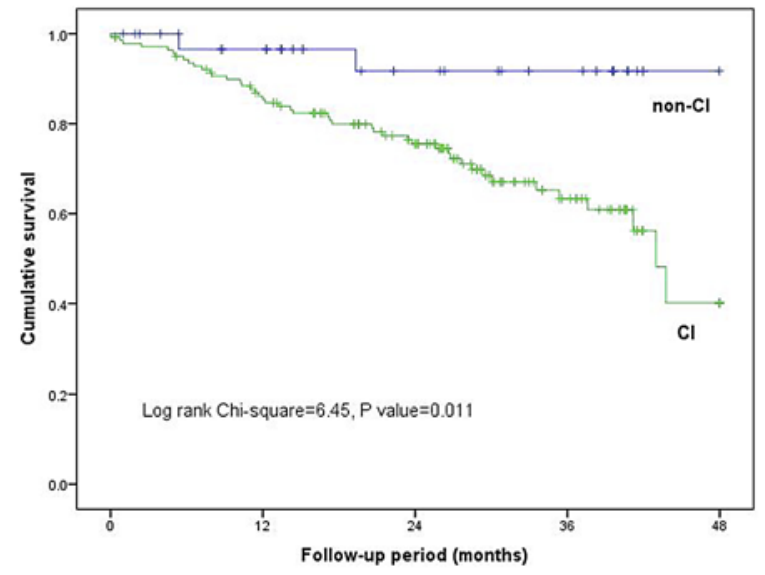

Number of patients at risk

$\begin{array}{lrrrrr}\text { Non-CI } & 34 & 26 & 17 & 12 & 0 \\ \text { CI } & 141 & 114 & 82 & 31 & 0\end{array}$

B

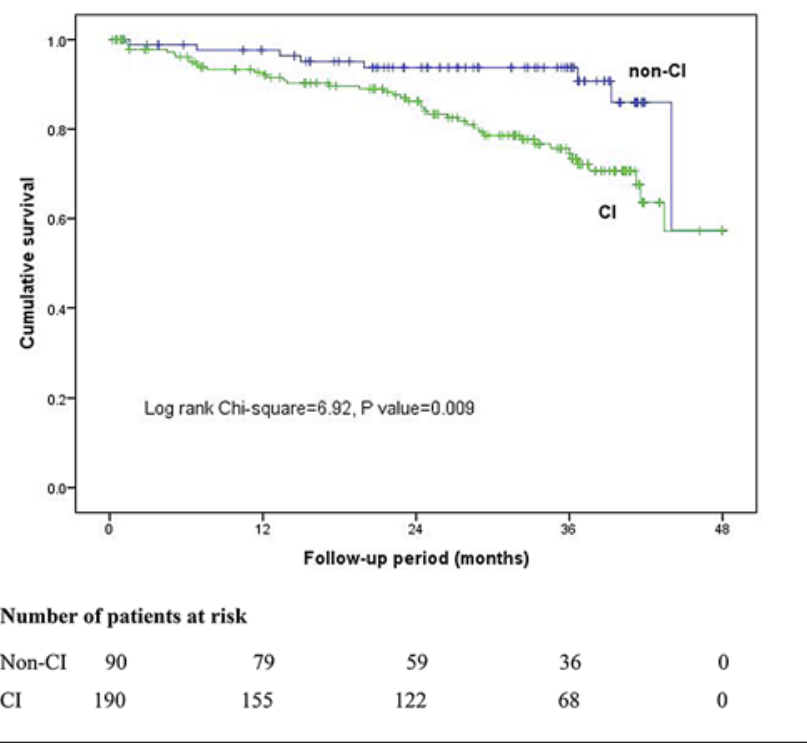

Fig. 2. Kaplan-Meier estimates of survival for patients with CI and without $C I$ in the DM (A) and non-DM (B) groups. CI, cognitive impairment; DM, diabetes mellitus.

Factors Associated with Cognitive Impairment in

Diabetic Patients Undergoing PD

Compared with diabetic PD patients without CI, those with CI had a lower level of education, and more of them need assisted PD (online suppl. Table 2). To analyze the risk factors related to $\mathrm{CI}$ in diabetic patients, variables that were considered clinically relevant to $\mathrm{CI}$ or with a $p$ value $<0.1$ in univariate tests were included in the multivariate model. In the multivariable regression analysis, the factors age, gender, education, BMI, history of smoking, CVD, MAP, total cholesterol, serum $\mathrm{Cr}$, and glucose were included, and a history of CVD (OR, 2.810; 95\% CI, 1.111-7.107) was associated with CI. In model 2, all the factors in model 1 plus $\mathrm{HbAlc}$ were included, and a history of CVD (OR, 2.926; 95\% CI, 1.139-7.516) and high HbA1c (OR, 1.547; 95\% CI, 1.013-2.362) were independently associated with a risk of CI (Table 3 ).

\section{Patient Outcomes}

By the end of the study, 73 (51.8\%) diabetic patients with CI remained on PD, 45 (31.9\%) died, 20 (14.2\%) were transferred to $\mathrm{HD}, 2$ (1.4\%) received kidney transplantation, and $1(0.7 \%)$ withdrew from PD for other reasons. While in diabetic patients without CI, 19 (55.9\%) remained on PD, 2 (5.9\%) died, 8 (23.5\%) were transferred to $\mathrm{HD}$, and $5(14.7 \%)$ received kidney transplanta- tion (online suppl. Table 3). During a median of 26.0 months (IQR 13.5-35.6 months) of follow-up period, 47 (26.9\%) diabetic patients died. Of these deaths, 28 (59.6\%) were caused by CVD, 8 (17.0\%) by infection, $3(6.4 \%)$ by malignancy, $3(6.4 \%)$ by cachexia, $2(4.3 \%)$ by other factors, and $3(6.4 \%)$ by an unknown reason. Except for 1 death caused by CVD and 1 by infection, the other 45 patients had CI. The Kaplan-Meier estimates of survival curves for patients with and without CI are shown in Figure 2. The log-rank test revealed that diabetic patients with $\mathrm{CI}$ had a lower rate of survival than those without CI $\left(\chi^{2}=6.45, p=0.011\right)$ (Fig. 2A). In the univariate Cox regression analyses, CI, advanced age, history of CVD, a longer duration of dialysis, lower mGFR, hemoglobin, HDL-C, and low-density lipoprotein cholesterol were significantly associated with all-cause mortality. And CI was an independent risk factor for mortality in diabetic patients after adjustment for age, CVD, dialysis vintage, and mGFR (HR, 7.224; 95\% CI, 1.694-30.806) (Table 4). Whereas in nondiabetic patients, CVD (HR, 1.915; 95\% CI, 1.093-3.356), instead of CI, was a significant risk factor for mortality after adjustment.

During the follow-up period, 61 episodes of peritonitis were observed in 40 diabetic PD patients (0.17 episodes per patient-year). The Poisson analysis showed that these DM patients with CI had a significantly lower incidence 
Table 4. Associations between cognitive impairment and mortality in diabetic patients by Cox regression analysis

\begin{tabular}{|c|c|c|c|c|}
\hline \multirow[t]{2}{*}{ Variable } & \multicolumn{2}{|l|}{ Univariate model } & \multicolumn{2}{|l|}{ Multivariate model } \\
\hline & HR (95\% CI) & $p$ value & $\operatorname{HR}(95 \% \mathrm{CI})$ & $p$ value \\
\hline Age, years & $1.075(1.040-1.111)$ & $<0.001$ & $1.079(1.042-1.117)$ & $<0.001$ \\
\hline Smoking & $0.869(0.482-1.567)$ & 0.641 & - & - \\
\hline CVD & $1.944(1.084-3.487)$ & 0.026 & $1.160(0.623-2.159)$ & 0.640 \\
\hline Cerebrovascular disease & $1.341(0.695-2.586)$ & 0.382 & - & - \\
\hline Dialysis vintage, month & $1.017(1.008-1.026)$ & $<0.001$ & $1.018(1.004-1.031)$ & 0.010 \\
\hline Assisted PD & $1.609(0.908-2.854)$ & 0.104 & - & - \\
\hline $\mathrm{mGFR}, \mathrm{ml} / \mathrm{min} / 1.73 \mathrm{~m}^{2}$ & $0.816(0.691-0.964)$ & 0.017 & $0.872(0.706-1.075)$ & 0.200 \\
\hline Hemoglobin, g/L & $0.986(0.974-0.999)$ & 0.033 & - & - \\
\hline $\mathrm{iPTH}, \mathrm{pg} / \mathrm{mL}$ & $1.001(1.000-1.001)$ & 0.051 & - & - \\
\hline $\mathrm{HDL}-\mathrm{C}, \mathrm{mmol} / \mathrm{L}$ & $0.221(0.075-0.651)$ & 0.006 & - & - \\
\hline $\mathrm{LDL}-\mathrm{C}, \mathrm{mmol} / \mathrm{L}$ & $0.628(0.434-0.909)$ & 0.014 & - & - \\
\hline Cognitive impairment & $5.185(1.256-21.399)$ & 0.023 & $7.224(1.694-30.806)$ & 0.008 \\
\hline
\end{tabular}

HR, hazard ratio; 95\% CI, 95\% confidence interval; CVD, cardiovascular disease; PD, peritoneal dialysis; mGFR, measured glomerular filtration rate; $\mathrm{iPTH}$, intact parathyroid hormone; HDL-C, high-density lipoprotein cholesterol; LDL-C, low-density lipoprotein cholesterol; MoCA, Montreal Cognitive Assessment.

of peritonitis ( 0.14 vs. 0.29 episodes per patient-year) than patients without CI. Compared to the diabetic PD patients without $\mathrm{CI}$, those with $\mathrm{CI}$ had a much higher proportion of assisted PD ( 44.7 vs. $17.6 \%, p<0.05$ ), although they were at a similar age. But this effect was not observed in non-DM patients. The patients with CI had a higher risk of peritonitis than those without CI ( 0.20 vs. 0.09 episodes per patient-year). During the follow-up period, 28 (16.0\%) diabetic patients were transferred to HD. There was no significant difference between the 2 groups with regard to technique survival.

\section{Discussion}

In the present study, we found the prevalence of CI to be $72.7 \%$ in the matched PD population, and this rate was up to $80.6 \%$ in diabetic patients. A significant association was found between DM and CI. The HbAlc level and CVD were independently associated with a risk of CI, and CI was an independent risk factor for mortality in diabetic patients undergoing PD. CI was also associated with a higher rate of peritonitis in nondiabetic patients but not in the diabetic population.

The prevalence of CI ranged from 17 to $62 \%$ in CKD patients reported by previous studies [1-3], and this rate reached as high as $87 \%$ in populations undergoing dialysis [4-6]. This discrepancy was probably due to differences in demographic characteristics, recruitment crite- ria, or the assessment methods used for CI. However, few studies have assessed CI in diabetic PD populations. In the present study, $72.7 \%$ of $\mathrm{PD}$ patients were identified as $\mathrm{CI}$, and we found that the prevalence was $80.6 \%$ in diabetic patients. Although they had a higher prevalence of CVD than nondiabetic patients, which are risk factors for $\mathrm{CI}$, the 2 groups were balanced in age, gender, MAP, education level, and hsCRP level after propensity score matching. The association between DM and CI remained significant after adjustment. Of the few studies in diabetic patients with CKD, Liao et al. [22] demonstrated that $35 \%$ of patients undergoing PD had CI, but the cognitive function was comparable between diabetic patients without retinopathy and nondiabetic patients. Another cohort study showed that $48 \%$ of diabetic patients with CKD stage 3 or 4 had neurocognitive disorders [23]. In the general population with DM, a longitudinal cohort study conducted in northern Manhattan reported that $42.5 \%$ of diabetic patients had incident CI [15]. Roberts et al. [16] found that $21.4 \%$ of patients with type 2 diabetes developed CI, despite the old age. There is a study that has found an interaction between diabetes and renal dysfunction in their effects on cognitive performance. Individuals with both diabetes and renal dysfunction are 4.23 times more likely to have CI than those with neither condition [24]. The potential explanation for this is that several mechanisms involved in CI also play roles in both diabetes and $\mathrm{CKD}$, such as vascular disease, inflammation, and oxidative stress $[25,26]$. Thus, the concurrence 
of diabetes and impaired renal function considerably increases the risk of CI.

Univariate and multivariate analyses of the present data have demonstrated that high $\mathrm{HbAlc}$ and a high prevalence of CVD are associated with a risk of CI in diabetic patients, suggesting that poor glycemic control and vascular dysfunction contribute to these cognitive deficits. The relationship between $\mathrm{HbA} 1 \mathrm{c}$ and cognitive function in diabetes has been well established. However, whether these associations exist in patients undergoing PD has rarely been explored. Seidel et al. [27] found that $\mathrm{HbAlc}$ was a risk factor for $\mathrm{CI}$ in $\mathrm{CKD}$ (including dialysis) patients, of whom $38.5 \%$ had diabetes. A large volume of the literature manifests that glycemia, especially $\mathrm{HbA} 1 \mathrm{c}$ concentration, correlates with cognitive function in diabetes [28]. Both high and low glucose concentrations (including random and fasting blood glucose, and glycated hemoglobin) are associated with a higher risk of dementia, even among people without diabetes [29, 30]. Glucose abnormalities might cause cerebrovascular disease, which is thought to be an important pathogenesis involved in cognitive decline [26]. In the present study, diabetic patients with CI did not show a higher prevalence of cerebrovascular disease than those without CI. Instead, CVD was an independent risk factor for $\mathrm{CI}$ in diabetic patients. It is well known that vascular risk factors are important for the development of cognitive impairment in the general population [31, 32]. Unfortunately, explorations regarding these factors and $\mathrm{CI}$ in patients with $\mathrm{CKD}$ or diabetes are scarce, and evidence is inconsistent. A crosssectional study of $\mathrm{HD}$ patients showed that CVD is associated with poorer executive function [33]. And the role of vascular factors in CI among diabetic patients has been demonstrated by limited research $[34,35]$. In view of the high burden of traditional cardiovascular risk factors in CKD and diabetic patients, these factors might also predispose diabetic CKD patients to CI. However, since targeting cardiovascular factors does not show a positive effect, uremic neurotoxins interacting with the nervous system are considered to be more important in CKD-associated CI $[9,32]$. Unexpectedly, age was not found to be a risk factor for $\mathrm{CI}$ in patients undergoing $\mathrm{PD}$ in the present study, unlike in previous studies. Since CI is an age-related comorbidity in the general population, commonly, old age of diabetic subjects may eliminate the difference.

There have been few investigations of clinical outcomes in PD patients relating to CI. Griva et al. [5] found a prevalence of $\mathrm{CI}$ in dialysis patients at baseline of $67.6 \%$ and a 2.5 -fold higher risk of mortality 7 years later. In ad- dition, Drew et al. [36] demonstrated that poor memory and executive function increase the risk of mortality in HD patients. These associations between CI and increased mortality have also been shown in other clinical populations and community cohorts $[37,38]$. Research conducted in patients with ESRD found dementia to be significantly associated with adverse clinical outcomes [39]. The Dialysis Outcomes and Practice Patterns Study (DOPPS) revealed that dementia is associated with a nearly 1.5-fold increased risk of death in HD patients [7]. But the relationship between mild CI and mortality was not evaluated. The present data indicated that diabetic patients with CI had a lower survival rate, which is consistent with the results of previous studies. Moreover, CI represented as an independent risk factor for mortality, rather than $C V D$, in patients with $\mathrm{DM}$, suggesting that it might be a more powerful factor than CVD to reflect systemic vascular dysfunction. Indeed, diabetic patients with CI were seven times more likely to die than patients without CI. Therefore, CI may represent a strong predictor of mortality in diabetic patients undergoing $\mathrm{PD}$, but further studies will be required to confirm these associations. In nondiabetic patients, however, CVD was still the predominant factor for patient survival, since the prevalence of CI was relatively lower in this population.

Another interesting finding was that diabetic patients without CI had a significantly higher incidence of peritonitis than patients with CI. There are several possible explanations for this finding. First, more of these patients performed their own $\mathrm{PD}$, rather than underwent assisted PD. For older people who have difficulty in peritoneal exchange due to physical disabilities or psychosocial problems, assisted PD is an alternative choice. CI was reported to be associated with $\mathrm{PD}$ assistance in patients older than 50 years [40]. Although patients on assisted PD might have shorter patient survival and peritonitis-free survival, they were usually older and had more comorbidities [41, 42]. Many studies also showed that PD assistance did not increase peritonitis risk or were associated with a lower risk of peritonitis in the elderly population $[42,43]$. In the current cohort, diabetic patients with different cognitive function were of similar age and had a comparable vascular profile and nutritional status. In addition, the caregivers have received standardized training and have been approved by the PD nurses. Due to advanced age and the burden of comorbidities, self-care PD may increase the risk of peritonitis alongside PD-related comorbidities in elderly patients during peritoneal exchanges. Thus, with adequate standardized training, assisted PD could protect diabetic patients from peritonitis. 
Second, patients without CI survived longer, meaning that it was possible for more episodes of peritonitis to occur. Third, because of the small sample size of patients without CI, the possibility of sampling bias cannot be excluded. Accordingly, patients with CI did not show a worse technique survival rate much likely due to the lower rate of peritonitis.

The present study had several limitations. First, as a single-center study, the small sample size and single ethnicity may limit its generalizability. Second, because less healthy patients and patients with severe CI were less likely to participate, the prevalence of CI was likely to have been underestimated. Third, only the MoCA was applied for cognitive assessment in the current study. This is a short screening instrument that cannot evaluate the cognitive function as thoroughly as comprehensive neuropsychological test batteries. Magnetic resonance imaging is also recommended to identify silent brain infarcts, microbleeds, and white matter disease. Another possible limitation is that DM-related confounders, such as medication and dialysate components, were not included due to incomplete data, which might have affected the impact of DM on CI.

In summary, we have found that the prevalence of CI is $72.7 \%$ in an age-, gender-, and MAP-matched CAPD population and $80.6 \%$ if these patients have DM. The association between DM and CI might at least in part be explained by high $\mathrm{HbAlc}$ and a higher risk of CVD, which provides a possible link with CKD-related CI. Diabetic patients with CI had a higher incidence of adverse outcomes. Moreover, CI, rather than CVD, was an independent risk factor for mortality in diabetic patients undergoing PD. For the fact that the underlying mechanisms and potential therapeutic targets for cognitive dysfunction are far from fully understood, the multitude of confounding factors connecting kidney and brain pathologies make the identification of associations difficult. Therefore, a large-scale longitudinal study to explore the causes and outcomes of CI should be performed in patients undergoing PD to determine the prognostic significance of these risk factors.

\section{Acknowledgements}

We thank all the doctors and nurses in our PD center for their patient care and data collection.

\section{Statement of Ethics}

All the subjects have given their written informed consent, and the study protocol was approved by the Human Ethics Committee of Sun Yat-sen University on human research (reference number [2016] 215).

\section{Conflict of Interest Statement}

The authors have no conflicts of interest to declare.

\section{Funding Sources}

The authors disclosed receipt of the following financial support for the research, authorship, and/or publication of this article: this work was supported by the National Natural Science Foundation of China $(81774069,81570614)$; the National Key Research and Development Program [2016YFC0906101]; the Special Fund for NHFPC Scientific Research in Public Welfare (201502023); the Natural Science Foundation of Guangdong Province, China (2014B020212020, 2017A050503003, and 2017B020227006); the Foundation of Guangdong Key Laboratory of Nephrology [2017B030314019]; and the Guangzhou Committee of Science and Technology, China (2014Y2-00543, 201704020167).

\section{Author Contributions}

All authors have contributed significantly and in keeping with the latest guidelines of the International Committee of Medical Journal Editors. Xiao Yang, Xuan Huang, and Chunyan Yi conceived and designed the study. Chunyan Yi, Yagui Qiu, Xiao Xi, and Jianxiong Lin prepared and performed data collection. Xuan Huang, Meiju $\mathrm{Wu}$, and Haishan $\mathrm{Wu}$ analyzed the data. Xuan Huang and Chunyan Yi interpreted the results and drafted the manuscript. Xiao Yang, Hongjian Ye, and Yuan Peng revised the manuscript. Xueqing Yu and Xiao Yang coordinated the study and finally approved the version to be published.

\section{References}

Cognitive Impairment in Diabetic

Patients Undergoing PD
1 Kurella M, Chertow GM, Luan J, Yaffe K. Cognitive impairment in chronic kidney disease. J Am Geriatr Soc. 2004;52(11):1863-9.

2 Rodriguez-Angarita CE, Sanabria-Arenas RM, Vargas-Jaramillo JD, Ronderos-Botero I. Cognitive impairment and depression in a population of patients with chronic kidney disease in Colombia: a prevalence study. Can J Kidney Health Dis. 2016;3:26.
3 Otobe Y, Hiraki K, Hotta C, Nishizawa H, Izawa KP, Taki Y, et al. Mild cognitive impairment in older adults with pre-dialysis patients with chronic kidney disease: prevalence and association with physical function. Nephrology (Carlton). 2019;24:50-5.

4 Murray AM, Tupper DE, Knopman DS, Gilbertson DT, Pederson SL, Li S, et al. Cognitive impairment in hemodialysis patients is common. Neurology. 2006;67(2):216-23. 
5 Griva K, Stygall J, Hankins M, Davenport A, Harrison M, Newman SP. Cognitive impairment and 7-year mortality in dialysis patients. Am J Kidney Dis. 2010;56(4):693-703.

6 Shea YF, Lam MF, Lee MS, Mok MY, Lui SL, Yip TP, et al. Prevalence of cognitive impairment among peritoneal dialysis patients, impact on peritonitis and role of assisted dialysis. Perit Dial Int. 2016;36(3):284-90.

7 Kurella M, Mapes DL, Port FK, Chertow GM. Correlates and outcomes of dementia among dialysis patients: the Dialysis Outcomes and Practice Patterns Study. Nephrol Dial Transplant. 2006;21(9):2543-8.

8 O'Lone E, Connors M, Masson P, Wu S, Kelly PJ, Gillespie D, et al. Cognition in people With end-stage kidney disease treated with hemodialysis: a systematic review and metaanalysis. Am J Kidney Dis. 2016;67:925-35.

9 Viggiano D, Wagner CA, Blankestijn PJ, Bruchfeld A, Fliser D, Fouque D, et al. Mild cognitive impairment and kidney disease: clinical aspects. Nephrol Dial Transplant. 2020;35(1):10-7.

10 Saran R, Robinson B, Abbott KC, Agodoa L, Bhave N, Bragg-Gresham J, et al. US renal data system 2017 annual data report: epidemiology of kidney disease in the United States. Am J Kidney Dis. 2018;71:A7.

11 Ye H, Cao P, Zhang X, Lin J, Guo Q, Mao H, et al. Serum magnesium and cardiovascular mortality in peritoneal dialysis patients: a 5 -year prospective cohort study. Br J Nutr. 2018;120(4):415-23.

12 Liu X, Huang R, Wu H, Wu J, Wang J, Yu X, et al. Patient characteristics and risk factors of early and late death in incident peritoneal dialysis patients. Sci Rep. 2016;6:32359.

13 Koekkoek PS, Kappelle LJ, van den Berg E, Rutten GE, Biessels GJ. Cognitive function in patients with diabetes mellitus: guidance for daily care. Lancet Neurol. 2015;14(3):329-40.

14 Zhang J, Chen C, Hua S, Liao H, Wang M, Xiong Y, et al. An updated meta-analysis of cohort studies: diabetes and risk of Alzheimer's disease. Diabetes Res Clin Pract. 2017; 124:41-7.

15 Luchsinger JA, Reitz C, Patel B, Tang MX, Manly JJ, Mayeux R. Relation of diabetes to mild cognitive impairment. Arch Neurol. 2007;64(4):570-5.

16 Roberts RO, Knopman DS, Geda YE, Cha RH, Pankratz VS, Baertlein L, et al. Association of diabetes with amnestic and nonamnestic mild cognitive impairment. Alzheimers Dement. 2014;10(1):18-26.

17 Watson PE, Watson ID, Batt RD. Total body water volumes for adult males and females estimated from simple anthropometric measurements. Am J Clin Nutr. 1980;33(1):2739.

18 American Psychiatric Association. Diagnostic and statistical manual of mental disorders. 5th ed. American Psychiatric Association; 2013.
19 Nasreddine ZS, Phillips NA, Bédirian V, Charbonneau S, Whitehead V, Collin I, et al. The Montreal Cognitive Assessment, MoCA: a brief screening tool for mild cognitive impairment. J Am Geriatr Soc. 2005;53(4):6959.

20 Abd RM, Ahmad NA, Chan YY, Mohamad KN, Yusof M, Abdul GM, et al. Validity of screening tools for dementia and mild cognitive impairment among the elderly in primary health care: a systematic review. Public Health. 2019;169:84-92.

21 Patnode CD, Perdue LA, Rossom RC, Rushkin MC, Redmond N, Thomas RG, et al. Screening for cognitive impairment in older adults: an evidence update for the U.S. preventive Services Task Force. Rockville (MD): Agency for Healthcare Research and Quality (US); 2020.

22 Liao JL, Xiong ZY, Yang ZK, Hao L, Liu GL, Ren YP, et al. An association of cognitive impairment with diabetes and retinopathy in end stage renal disease patients under peritoneal dialysis. PLOS ONE. 2017;12(8): e0183965.

23 Hobson P, Lewis A, Nair H, Wong S, Kumwenda $\mathrm{M}$. How common are neurocognitive disorders in patients with chronic kidney disease and diabetes? Results from a cross-sectional study in a community cohort of patients in North Wales, UK. BMJ Open. 2018; 8(12): 023520.

24 Yin Z, Yan Z, Liang Y, Jiang H, Cai C, Song A, et al. Interactive effects of diabetes and impaired kidney function on cognitive performance in old age: a population-based study. BMC Geriatr. 2016;16:7.

25 Bronas UG, Puzantian H, Hannan M. Cognitive impairment in chronic kidney disease: vascular Milieu and the potential therapeutic role of exercise. Biomed Res Int. 2017;2017: 2726369.

26 McCrimmon RJ, Ryan CM, Frier BM. Diabetes and cognitive dysfunction. Lancet. 2012; 379(9833):2291-9.

27 Seidel UK, Gronewold J, Volsek M, Todica O, Kribben A, Bruck H, et al. The prevalence, severity, and association with $\mathrm{HbAlc}$ and fibrinogen of cognitive impairment in chronic kidney disease. Kidney Int. 2014;85(3):693702.

28 Geijselaers SLC, Sep SJS, Stehouwer CDA, Biessels GJ. Glucose regulation, cognition, and brain MRI in type 2 diabetes: a systematic review. Lancet Diabetes Endocrinol. 2015;3(1):75-89.

29 Crane PK, Walker R, Hubbard RA, Li G, Nathan DM, Zheng $\mathrm{H}$, et al. Glucose levels and risk of dementia. N Engl J Med. 2013;369(6): 540-8.

30 Ravona-Springer R, Moshier E, Schmeidler J, Godbold J, Akrivos J, Rapp M, et al. Changes in glycemic control are associated with changes in cognition in non-diabetic elderly. J Alzheimers Dis. 2012;30(2):299-309.
31 de Bruijn RF, Bos MJ, Portegies ML, Hofman A, Franco OH, Koudstaal PJ, et al. The potential for prevention of dementia across two decades: the prospective, population-based Rotterdam Study. BMC Med. 2015;13:132.

32 Viggiano D, Wagner CA, Martino G, Nedergaard M, Zoccali C, Unwin R, et al. Mechanisms of cognitive dysfunction in CKD. Nat Rev Nephrol. 2020;16(8):452-69.

33 Weiner DE, Scott TM, Giang LM, Agganis BT, Sorensen EP, Tighiouart H, et al. Cardiovascular disease and cognitive function in maintenance hemodialysis patients. Am J Kidney Dis. 2011;58(5):773-81.

34 Feinkohl I, Price JF, Strachan MW, Frier BM. The impact of diabetes on cognitive decline: potential vascular, metabolic, and psychosocial risk factors. Alzheimers Res Ther. 2015; 7(1):46.

35 Haroon NN, Austin PC, Shah BR, Wu J, Gill SS, Booth GL. Risk of dementia in seniors with newly diagnosed diabetes: a populationbased study. Diabetes Care. 2015;38(10): 1868-75.

36 Drew DA, Weiner DE, Tighiouart $\mathrm{H}$, Scott $\mathrm{T}$, Lou K, Kantor A, et al. Cognitive function and all-cause mortality in maintenance hemodialysis patients. Am J Kidney Dis. 2015;65(2): 303-11.

37 Hapca S, Guthrie B, Cvoro V, Bu F, Rutherford AC, Reynish E, et al. Mortality in people with dementia, delirium, and unspecified cognitive impairment in the general hospital: prospective cohort study of 6,724 patients with 2 years follow-up. Clin Epidemiol. 2018; 10:1743-53.

38 An R, Liu GG. Cognitive impairment and mortality among the oldest-old Chinese. Int J Geriatr Psychiatry. 2016;31(12):1345-53.

39 Thamer M, Kaufman JS, Zhang Y, Zhang Q, Cotter DJ, Bang H. Predicting early death among elderly dialysis patients: development and validation of a risk score to assist shared decision making for dialysis initiation. Am J Kidney Dis. 2015;66(6):1024-32.

40 Farragher JF, Oliver MJ, Jain AK, Flanagan S, Koyle K, Jassal SV. PD assistance and relationship to co-existing geriatric syndromes in incident peritoneal dialysis therapy patients. Perit Dial Int. 2019 Jul-Aug;39(4):375-81.

$41 \mathrm{Ng} \mathrm{JK}$, Chan GC, Chow KM, Fung W, Pang WF, Law MC, et al. Helper-assisted continuous ambulatory peritoneal dialysis: does the choice of helper matter?. Perit Dial Int. 2020; 40(1):34-40.

42 Castrale C, Evans D, Verger C, Fabre E, Aguilera D, Ryckelynck JP, et al. Peritoneal dialysis in elderly patients: report from the French Peritoneal Dialysis Registry (RDPLF). Nephrol Dial Transplant. 2010;25(1):255-62.

43 Duquennoy S, Béchade C, Verger C, Ficheux M, Ryckelynck JP, Lobbedez T. Is peritonitis risk increased in elderly patients on peritoneal dialysis? Report from the French Language Peritoneal Dialysis Registry (RDPLF). Perit Dial Int. 2016;36(3):291-6. 